\title{
Classification Identification of Acoustic Emission Signals from Underground Metal Mine Rock by ICIMF Classifier
}

\author{
Hongyan Zuo, ${ }^{1,2} \mathrm{Zhouquan} \mathrm{Luo,}^{1}$ and Chao $\mathrm{Wu}^{1}$ \\ ${ }^{1}$ School of Resource and Safety Engineering, Central South University, Changsha 410083, China \\ ${ }^{2}$ Commercial College, Hunan International Economics University, Changsha 410205, China \\ Correspondence should be addressed to Hongyan Zuo; zuohongyan18@126.com
}

Received 27 June 2014; Accepted 7 October 2014; Published 21 October 2014

Academic Editor: Peng-Yeng Yin

Copyright (c) 2014 Hongyan Zuo et al. This is an open access article distributed under the Creative Commons Attribution License, which permits unrestricted use, distribution, and reproduction in any medium, provided the original work is properly cited.

\begin{abstract}
To overcome the drawback that fuzzy classifier was sensitive to noises and outliers, Mamdani fuzzy classifier based on improved chaos immune algorithm was developed, in which bilateral Gaussian membership function parameters were set as constraint conditions and the indexes of fuzzy classification effectiveness and number of correct samples of fuzzy classification as the subgoal of fitness function. Moreover, Iris database was used for simulation experiment, classification, and recognition of acoustic emission signals and interference signals from stope wall rock of underground metal mines. The results showed that Mamdani fuzzy classifier based on improved chaos immune algorithm could effectively improve the prediction accuracy of classification of data sets with noises and outliers and the classification accuracy of acoustic emission signal and interference signal from stope wall rock of underground metal mines was $90.00 \%$. It was obvious that the improved chaos immune Mamdani fuzzy (ICIMF) classifier was useful for accurate diagnosis of acoustic emission signal and interference signal from stope wall rock of underground metal mines.
\end{abstract}

\section{Introduction}

The mining damage of underground surrounding rock was caused by mining, changing the balance of underground rock $[1,2]$; it was a common disaster that the impact of mining led to the occurrence of rock burst in underground works. Because evolution and formation of rock burst were a nonlinear process, the nonlinearity was the root cause of rock burst [3-6]. As shown in literature [3], the severities of roof fall accidents had been attempted to predict based on some major contributing parameters using the binary logistic regression model. The results revealed that wider gallery width was more prone to major and serious accidents than narrower gallery width. As shown in literature [4], Liu et al. had divided the roof caving hidden danger of roof area by roof structure detection instrumentation and the results showed that the division hidden danger of roof caving method based on the roof structure detection and peek into the roof had a higher similar rate to underground roof strata conditions. As shown in literature [5], rock samples were collected from horizontal stress-related roof fall material in coal mines for petrographic characterization and compressive strength testing and the results revealed that the great variability of strength, texture, and mineralogy documented in these samples may be an indication of their complexity and the need for specialized methodology in the study of shale strength. As shown in literature [6], a relative risk model for roof and side fall fatal accidents was developed using log-linear analysis of two-way contingency table. A few statistics such as potential fatalities $(\mathrm{PF})$, relative risk of fatalities (RRF), and safety measure effectiveness (SME) were derived which can be used as key safety performance indicators of roof and side fall accidents in underground mines. The application reveals that the model and the statistics developed in this study were generic and can be applied to any industrial setting.

Thus, the view of traditional deterministic was used to study mine roof fall which was a nonlinear dynamics process, as a stationary view was used to look at the evolving and changing problems [7-10]; Bobick et al. [7] had used a weighted manikin mounted to generated against the top rail and nine construction workers, who served as test subjects; each built five different guardrail configurations. The study 
results revealed that it was possible and effective methods for evaluation of guardrail systems for preventing falls through roof and floor holes. As shown in literature [8], fuzzy classification using features selected for computational ease was applied to the mine data. Both large roof fall events could be predicted successfully using a roof fall index (RFI) metric calculated from the results of the fuzzy classification. Zheng and Cai [9] had introduced an ANN subsystem to ensure technological and scale efficiencies of the training samples for ANN. The results revealed that the ANN subsystem was proved to be very efficient. As shown in literature [10], an extension to an established theory of cutoff grade was proposed herein to determine the optimal production capacities based on a stochastic framework relying on multiple grade-tonnage curves derived from a set of simulated orebody realizations. Application to an actual copper deposit demonstrates the benefits of the proposed model. The error of understanding and prediction even error were inevitable. Much information contained in the process of rock burst in underground metal mines was reflected by rock acoustic emission phenomenon [1]. By analyzing acoustic emission characteristics of rock rupture process in underground metal mines to identify rock fracture mechanism, theoretical basis would be provided for the acoustic emission monitoring techniques of underground metal mines rock. As a variety of sound and vibration led by production operations of the underground metal mine, such as drilling operations, blasting operations, locomotive motion, unloading ore, and ore drawing [11], these sounds or vibrations were easily confused with acoustic emission signals in rock fracture process; so the validity and accuracy of the acoustic emission monitoring results were greatly affected [12]; multiangle comparative study on acoustic emission characteristics of underground metal mines and multifeature integrated recognition technology theory which was different from the noise signal were still worthy of further discussion and study.

Therefore, in the paper, it was proposed that bilateral Gaussian membership function parameters were made to be constraints; the validity index [13] and the corrected sample number [14] of fuzzy classification were made to be subobjective of the fitness function; Mamdani fuzzy classifier using improved chaos immune algorithm [15-17] to optimize was established; the disadvantage which was adjusting the weights of rule to change the parameters of fuzzy systems was avoided; good technical support and theoretical support for the monitoring signal classification identification of underground metal mines rock were provided.

\section{Design of Mamdani Fuzzy Classifier Based on Improved Chaos Immune Algorithm}

2.1. Establishment of Mamdani Fuzzy Classifier. For Mamdani fuzzy classifier with $n$ classified input samples and $m$ rules, Mamdani fuzzy rule can be expressed by (1) as follows:

$$
\begin{aligned}
& R_{k}: \text { If } x_{1 k} \text { was } \mu_{1 k}, x_{2 k} \text { was } \mu_{2 k}, \ldots, \\
& \quad x_{n k} \text { was } \mu_{n k} \text {, then } y_{k} \text { was } \mu\left(n+1, c_{k}\right) \text {, }
\end{aligned}
$$

where $\mu_{j k}$ was the membership of input sample $x_{j}(j=$ $1,2, \ldots, n)$ to be classified under the $k$ th rule; $y_{k}(k=$ $1,2, \ldots, m)$ was the classified output variable under the $k$ th rule; $c_{k}$ was the output category number of Mamdani fuzzy classifier under the $k$ th rule.

To reach a better statistical property, Gaussian membership function with statistical nature was taken as the membership function of rule antecedent; that is, bilateral Gaussian membership function was used to represent the membership function of the $j$ th variable $x_{j k}$ corresponding to the $k$ th rule as

$$
\mu_{j k}= \begin{cases}\exp \left[-\frac{x_{j k}-\delta_{j k}}{\alpha_{j k}}\right]^{2} & x_{j k} \leq \delta_{j k} \\ \exp \left[-\frac{x_{j k}-\delta_{j k}}{\beta_{j k}}\right]^{2} & x_{j k}>\delta_{j k},\end{cases}
$$

where $\delta_{j k}(j=1,2, \ldots, n), \alpha_{j k}(j=1,2, \ldots, n)$, and $\beta_{j k}(j=$ $1,2, \ldots, n)$ denote the midpoint, the left width, and the right width of bilateral Gaussian membership function of the $j$ th variable corresponding to the $k$ th rule.

To make Mamdani fuzzy classifier have better classification capability, single point membership function was adopted for the consequence of fuzzy rule as follows:

$$
\mu\left(n+1, c_{k}\right)= \begin{cases}1 & y=c_{k} \\ 0 & y \neq c_{k} .\end{cases}
$$

Assuming the total number of categories was $M$, it can be known from the experiment that the output domain of Mamdani fuzzy logic system was $[1, M]$, which was divided into $M$ areas averagely and each area was a category. The output category of Mamdani fuzzy classifier can be calculated through (4) as

$$
c_{k}=\operatorname{int}\left[\frac{M}{M-1}(\omega-1)\right],
$$

where $\omega$ was the output of Mamdani fuzzy logic system and $M$ was the total number of sample categories.

2.2. Indexes of Fuzzy Classification Effectiveness. For $\mathbf{X}=$ $\left\{x_{1}, x_{2}, \ldots, x_{n}\right\}$, the cut set $d_{k}$ of fuzzy class $c_{k}$ under the $k$ th rule was set as

$$
\begin{aligned}
d_{k} & =\left\{d_{1 k}, d_{2 k}, \ldots, d_{s k}\right\} \\
& =\left\{x_{k} \mid x_{k} \in X, \text { and } k=\underset{1 \leq k \leq c}{\arg \max }\left\{\mu_{k}\left(x_{k}\right)\right\}\right\},
\end{aligned}
$$

where $\arg \max \left[\mu_{k}\left(x_{k}\right)\right]$ was the fuzzy class $c_{k}$ which was assigned to the $k$ th rule under mode $x$ according to the principle of maximum membership; $s$ was the number of effective samples under the $k$ th rule.

Then, using the cut set of fuzzy class $c_{k}$ corresponding to the $k$ th rule and fuzzy membership matrix $\mathbf{U}$, the probability $P\left(c_{k}\right)$ of the $k$ th fuzzy class can be calculated by

$$
P\left(c_{k}\right)=\frac{\sum_{d_{j k} \in d_{k}} \mu_{k}\left(d_{j k}\right)}{\sum_{k=1}^{c} \sum_{d_{j k} \in d_{k}} \mu_{k}\left(d_{j k}\right)},
$$

where $c$ was the number of fuzzy classification. 
Probability $P\left(d_{j k}\right)$ of mode $d_{j k}$ can be expressed as

$$
P\left(d_{j k}\right)=\sum_{k=1}^{c} P\left(c_{k}\right) \mu_{k}\left(d_{j k}\right)
$$

The probability $P\left(c_{k} \mid d_{k}\right)$ of the cut set $d_{k}$ of fuzzy class $c_{k}$ corresponding to the $k$ th rule can be expressed as

$$
P\left(c_{k} \mid d_{k}\right)=\prod_{j=1}^{s} \frac{P\left(c_{k}\right) \mu_{k}\left(d_{j k}\right)}{P\left(d_{j k}\right)} .
$$

The occurring probability of fuzzy classification can be measured through the mean value of all the occurring probabilities of cut sets of fuzzy classes; then, the index $f(\mathbf{U}, c)$ of fuzzy classification effectiveness can be expressed as

$$
f(\mathbf{U}, c)=\sum_{k=1}^{c} \prod_{j=1}^{s} \frac{P\left(c_{k}\right) \mu_{k}\left(d_{j k}\right)}{\sum_{i=1}^{c} P\left(c_{k}\right) \mu_{k}\left(d_{j k}\right)} .
$$

The greater the value of $f(\mathbf{U}, c)$ was, the greater occurring probability of this fuzzy classification was. When its value reaches the maximum, the classification number it corresponds to was the optimal number $c^{*}$ of data set.

\subsection{Optimization of the Fitness Function by Improved Chaos} Immune Algorithm. The midpoint $\delta_{j k}$, left width $\alpha_{j k}$, and right width $\beta_{j k}$ of the bilateral Gaussian membership function of Mamdani fuzzy classifier can be optimized by improved chaos immune algorithm. Considering that the key to improved chaos immune algorithm was to determine the fitness function, in this study, the fitness function was expressed as follows:

$$
F\left(\delta_{i k}, \alpha_{i k}, \beta_{i k}\right)=w_{1} \frac{f(\mathbf{U}, c)}{f_{0}(\mathbf{U}, c)}+w_{2} \frac{N}{N_{0}}
$$

where $f_{0}(\mathbf{U}, c)$ was the initial value of fuzzy classification effectiveness index before optimization, $N$ was the number of samples which were fuzzy classified correctly after optimization, and $N_{0}$ was the number of samples with correct fuzzy classification before optimization. $w_{1}$ was the weight coefficient corresponding to the index of fuzzy classification effectiveness, $w_{2}$ was the weight coefficient corresponding to the number of samples which were fuzzy classified correctly, and $w_{1}+w_{2}=1$.

Figure 1 showed flow chart of optimizing parameters of Mamdani fuzzy classifier using improved chaos immune algorithm, and the specific steps were expressed as follows.

Step 1. Input $k$ th antigens $\left\{A_{g 1 k}, A_{g 2 k}, \ldots, A_{g l k}, \ldots, A_{g L k}\right\}$ $(k=1,2, \ldots, m)$ and carry out the standardization process.

Step 2. Select the logistic model $x_{r+1}=4 x_{r}\left(1-x_{r}\right)$ as $R$ chaotic variables of the $k$ th initialization antibodies $\left\{A_{b 1 k}, A_{b 2 k}, \ldots, A_{b r k}, \ldots, A_{b R k}\right\}(k=1,2, \ldots, m)$ in interval $(0,1)$, which were generated randomly by chaotic model.
Step 3. For each antigen $A_{\text {glk }}(l=1,2, \ldots, L ; k=$ $1,2, \ldots, m)$, it was operated as follows.

Step 3.1. Calculate the affinity $\beta_{r l}$ of each antibody $A_{b r k}$ and antigen $A_{\text {glk }}$, respectively, using (11) as follows:

$$
\beta_{r l}=\sqrt{\sum_{k=1}^{m}\left(A_{b r k}-A_{g_{l k}}\right)^{2}} .
$$

Step 3.2. Select $R_{0}$ antibodies with highest affinity as network cells and clone them and then obtain the corresponding number of clonal antibody cells $C_{0}$.

Step 3.3. For the clonal cells, equation $C_{i+1}=C_{i}-\alpha\left(C_{i}-X\right)$ ( $i=1,2, \ldots, R_{0}$ ) was used for their mutation, where $C_{i}$ was the number of clonal antibody cells, $X_{0}$ was the number of clonal antigen cells, and $\alpha$ was the mutation rate.

Step 3.4. Recalculate the affinity $\beta_{r l}$ of each antibody $A_{b r k}$ and antigen $A_{\text {glk }}$ after mutation using (11).

Step 3.5. Select $30 \%$ of the cells with highest affinity as the memory cell data set $M_{p}$.

Step 3.6. Calculate the similarity degree $\lambda_{r(R-r)}(r \neq R-r)$ between each antibody $A_{b r}$ and $A_{b(R-r)}$ using (12); eliminate the individuals whose similarity degree $\lambda_{r(R-r)}$ was greater than the threshold value $\sigma_{s}$ in the data set $M_{p}$ as follows:

$$
\lambda_{r(R-r)}=\sqrt{\sum_{k=1}^{m}\left[A_{b r k}-A_{b(R-r) k}\right]^{2}} .
$$

Step 4. Insert the memory cell data set $M_{p}$ into the memory dataset $M$.

Step 5. Select better antibody and antigen individuals for chaotic search.

Select $10 \%$ of the individuals whose fitness value was relatively large for chaotic fine search and set optimum individual as $T=\left(T_{1}, T_{2}, \ldots, T_{k}\right)$; the search interval of chaotic variables was narrowed as follows:

$$
\begin{aligned}
& a_{i}^{\prime}=T_{i}-\phi\left(b_{i}-a_{i}\right) \\
& b_{i}^{\prime}=T_{i}+\phi\left(b_{i}-a_{i}\right),
\end{aligned}
$$

where $\phi$ was the shrinkage factor and $\phi \in(0,0.5)$.

To ensure that the new range is not out of bound, deal with it as follows: if $a_{i}^{\prime}<a_{i}, a_{i}^{\prime}=a_{i}$; if $b_{i}^{\prime}>b_{i}, b_{i}^{\prime}=b_{i}$.

Therefore, vector $Y_{i}$ of $T_{i}$ after reduction in the new interval $\left[a_{i}^{\prime}, b_{i}^{\prime}\right]$ can be determined by (14) as follows:

$$
Y_{i}=\frac{T_{i}-a_{i}^{\prime}}{b_{i}^{\prime}-a_{i}^{\prime}} .
$$

Set the linear combination of $Y_{i}$ and $T_{i, n+1}$ as a new chaotic variable and use it for search. Consider

$$
T_{i, n+1}^{\prime}=\left(1-\delta_{i}\right) Y_{i}+\delta_{i} T_{i, n+1},
$$

where $\delta_{i}$ was the adaptive control coefficient and $0<\delta_{i}<1$. 


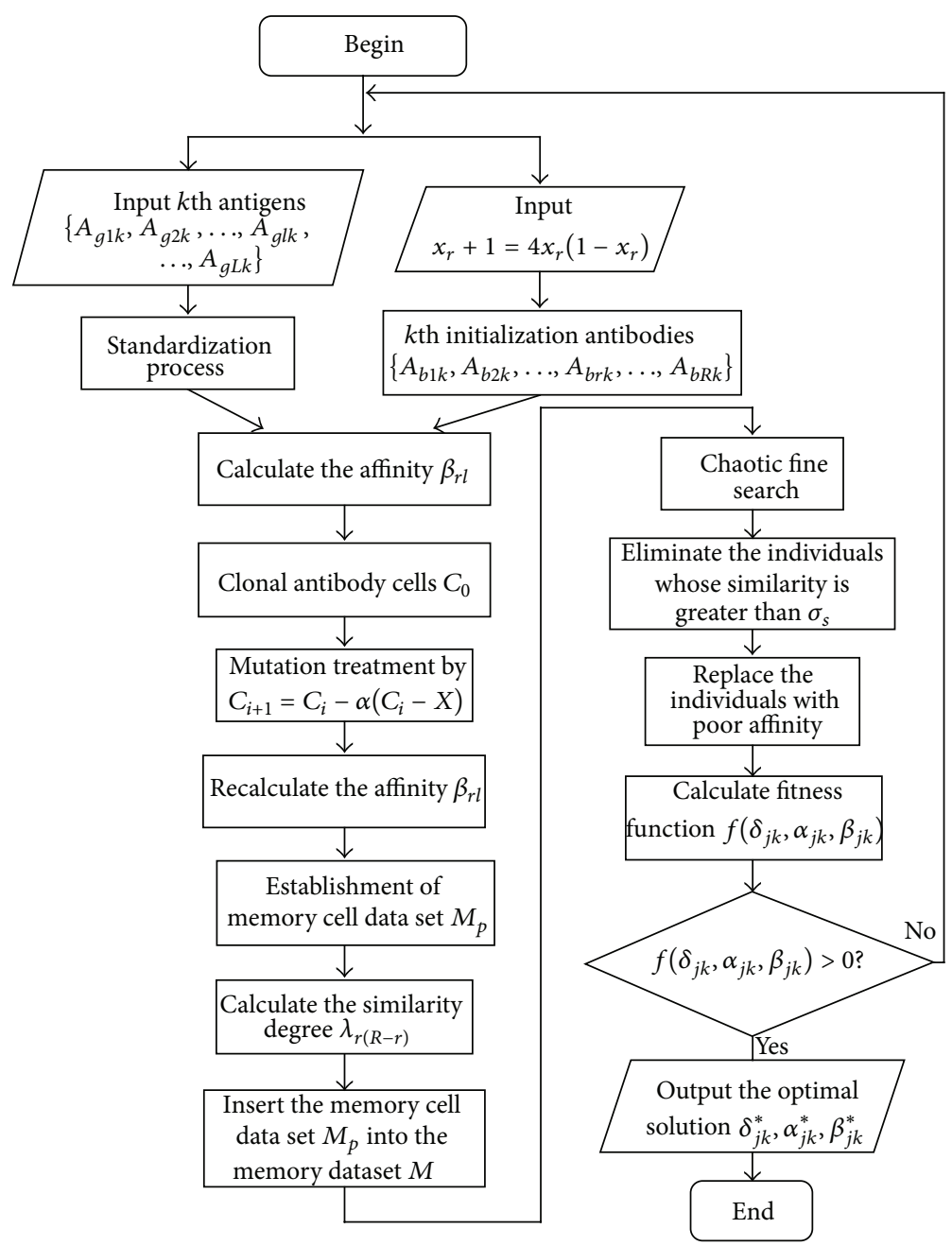

FIGURE 1: Flow chart of optimizing parameters of Mamdani fuzzy classifier using improved chaos immune algorithm.

Adaptive control coefficient $\delta_{i}$ was determined adaptively as follows:

$$
\delta_{i}=1-\left(\frac{K-1}{K}\right)^{\theta}
$$

where $\theta$ was a positive integer and was determined according to the objective function (in this paper, it was taken as 2.5); $K$ was the evolutional generation.

Eliminate the individuals whose similarity was greater than $\sigma_{s}$ in $10 \%$ of individuals with larger fitness value in the memory base.

Step 6. Select $x_{r+1}=4 x_{r}\left(1-x_{r}\right)$ for generating $N^{\prime}$ individuals in $(0,1)$ to replace the individuals with poor affinity; then, they were set as the antibodies for next immune computing together with the memory dataset $M_{p}$ which was obtained from last immune computing; return to Step 3 until the network reaches convergence.

Step 7. Use the fitness function $f\left(\delta_{j k}, \alpha_{j k}, \beta_{j k}\right)$ to evaluate $\delta_{j k}^{*}, \alpha_{j k}^{*}, \beta_{j k}^{*}$ and calculate the corresponding $f\left(\delta_{j k}^{*}, \alpha_{j k}^{*}, \beta_{j k}^{*}\right)$; if $f\left(\delta_{j k}^{*}, \alpha_{j k}^{*}, \beta_{j k}^{*}\right)>f\left(\delta_{j k}, \alpha_{j k}, \beta_{j k}\right)$, then $f\left(\delta_{j k}, \alpha_{j k}, \beta_{j k}\right)=$ $f\left(\delta_{j k}^{*}, \alpha_{j k}^{*}, \beta_{j k}^{*}\right)$; or else, abandon $\delta_{j k}^{*}, \alpha_{j k}^{*}, \beta_{j k}^{*}$.

Step 8. If the fitness function was the maximum which was greater than 1.0, then stop searching and output the optimal solution $\delta_{j k}^{*}, \alpha_{j k}^{*}, \beta_{j k}^{*}$; otherwise, return to Step 1 .

\subsection{Simulation Experiment of Mamdani Fuzzy Classifier} Based on Improved Chaos Immune Algorithm. To validate the robustness of the Mamdani fuzzy classifier (denoted by $\mathrm{C1}$ ) based on improved chaos immune algorithm to noises and outliers, Iris database was used for simulation experiment and its classification result was compared with those of the Mamdani fuzzy classifier (denoted by C2) in [14] based on improved genetic algorithm and the classifier (denoted by C3) in [18].

150 groups of Iris data were a very typical classification data proposed by the famous British statistician Fisher R. A. and can be used as evaluation criteria of various classification algorithms. Iris data was composed of 150 fourdimensional (pental length, pental width, sepal length, and 
TABLE 1: Parameter values of the optimized three kinds of fuzzy classifiers.

\begin{tabular}{lccccc}
\hline Classifier & Rule & $\delta_{j k}$ & $\alpha_{j k}$ & $\beta_{j k}$ & Category \\
\hline \multirow{3}{*}{ C1 } & 1 & 0.5844 & 0.4218 & 0.6656 & 1 \\
& 2 & 1.367 & 0.6789 & 0.9706 & 2 \\
& 3 & 2.4655 & 0.8996 & 0.1646 & 3 \\
\hline \multirow{3}{*}{ C2 } & 1 & 0.5732 & 0.4312 & 0.6472 & 1 \\
& 2 & 1.283 & 0.6472 & 0.9493 & 2 \\
& 3 & 2.325 & 0.9493 & 0.1592 & 3 \\
\hline \multirow{2}{*}{ C3 } & 1 & 0.5823 & 0.4224 & 0.6627 & 1 \\
& 2 & 1.335 & 0.6753 & 0.9675 & 2 \\
& 3 & 2.452 & 0.8956 & 0.1629 & 3 \\
\hline
\end{tabular}

TABLE 2: Comparison of classification accuracy of three kinds of fuzzy classifiers.

\begin{tabular}{lccc}
\hline Name of classifier & C1 & C2 & C3 \\
\hline Number of variables & 1 & 1 & 2 \\
Number of rules & 3 & 3 & 12 \\
Number of samples correctly classified & 147 & 144 & 146 \\
\hline Classification accuracy/\% & 98.00 & 96.00 & 97.33 \\
\hline
\end{tabular}

sepal width) samples and consists of a total of three categories (1-Iris-setosa, 2-Iris-versicolor, and 3-Iris-virginica), with 50 samples in each category. Category 1 was completely separate from the other 2 categories, while some cross exists between Category 2 and Category 3.

Parameters $\delta_{j k}, \alpha_{j k}$, and $\beta_{j k}$ of fuzzy classifiers C1, C2, and C3 after optimization were shown in Table 1 and the classification accuracy was compared in Table 2.

The results in Table 2 indicate that, for fuzzy classifier C3 after optimization, the number of variables was 2, the number of rules was 12 , the number of samples correctly classified was 146, that is, the number of samples misclassified was 4 , and the classification accuracy was $97.33 \%$; for fuzzy classifier C2 after optimization, the number of variables was 1 , the number of rules was 3 , the number of samples correctly classified was 144, that is, the number of samples misclassified was 6 , and the classification accuracy was $96.00 \%$; for fuzzy classifier $\mathrm{Cl}$ after optimization, the number of variables was 1 , the number of rules was 3 , the number of samples correctly classified was 147 , that is, the number of samples misclassified was 3 , and the classification accuracy was $98.00 \%$. It can be concluded that the Mamdani fuzzy classifier based on improved chaos immune algorithm proposed in this paper has higher classification accuracy for Iris data.

The computational complexity measured by CPU time was also compared with three kinds of fuzzy classifiers. As shown in Table 3, compared with the CPU time of the fuzzy classifier $\mathrm{C} 2$ and the fuzzy classifier $\mathrm{C} 3$, the $\mathrm{CPU}$ time of the fuzzy classifier $\mathrm{Cl}$ was the shortest. Obviously, it was easy to simply conclude that the fuzzy classifier $\mathrm{Cl}$ was less computationally expensive than the fuzzy classifier $\mathrm{C} 2$ or the fuzzy classifier C3.
TABLE 3: The CPU time of three kinds of the fuzzy classifiers.

\begin{tabular}{lccc}
\hline Data & \multicolumn{3}{c}{ CPU time $(\mathrm{s})$} \\
& $\mathrm{C} 1$ & $\mathrm{C} 2$ & $\mathrm{C} 3$ \\
\hline SPECTF & 0.007631 & 0.315737 & 0.016842 \\
Iris & 0.002986 & 0.015712 & 0.005712 \\
Lymphography & 0.005756 & 0.139592 & 0.010951 \\
Heart-disease-cleverland & 0.016427 & 0.312738 & 0.015944 \\
\hline Pendigits (test) & 0.275628 & 3.127292 & 0.535523 \\
\hline
\end{tabular}

TABLE 4: Classified results of measurement data ${ }^{1}$.

\begin{tabular}{lcccc}
\hline Classifier & \multicolumn{3}{c}{ Number of misclassified samples } & Classification \\
& F1 & F2 & F3 & accuracy/\% \\
\hline C1 & 3 & 4 & 4 & 81.67 \\
C2 & 2 & 3 & 3 & 86.67 \\
C3 & 2 & 2 & 2 & 90.00 \\
C3 & 2 & 2 & 2 & 90.00 \\
\hline
\end{tabular}

${ }^{1}$ F1-Rock blasting signals; F2-mechanical vibration signals; F3-acoustic emission signals.

\section{Practical Application of Mamdani Fuzzy Classifier Based on Improved Chaos Immune Algorithm}

Interference signals of acoustic emission signals from stope wall rock of underground metal mines mainly include mechanical vibration and blasting signals [1]. Figure 2 shows the test data of mechanical vibration, blasting signals, and acoustic emission signals from stope wall rock collected during the exploitation process of underground metal mines, and each consists of 500 groups. 120 effective sample data were fetched from acoustic emission signals and interference signals from stope wall rock of underground metal mines, 60 of which were set as the training set (20 rock blasting signals, 20 mechanical vibration signals, and 20 acoustic emission signals) and 60 as the sample test set $(20$ rock blasting signals $(\mathrm{mV}), 20$ mechanical vibration signals $(\mathrm{mV})$, and 20 acoustic emission signals $\left(10^{3} \mathrm{mV}^{2} \mathrm{~s}^{-1}\right)$ ). Classifier $\mathrm{C} 2$, classifier $\mathrm{C} 3$, and classifier $\mathrm{C} 1$ proposed in this paper were used, respectively, for the classification of test data of acoustic emission signals and interference signals from stope wall rock of underground metal mines and the results were shown in Table 4, from which it can be learned that the classification accuracy when using classifier C2, classifier C3, and classifier $\mathrm{Cl}$ proposed in this study is $81.67 \%, 86.67 \%$, and $90.00 \%$, respectively. It was thus clear that Mamdani fuzzy classifier optimized by improved chaos immune algorithm makes different samples have different contributions and, to a large extent, reduces the influence of noises and outliers on classification, making the learning algorithm more robust in the case of sensitive data or noisy data, for the indexes of fuzzy classification effectiveness and correct sample number of fuzzy classification were set as the subgoal of fitness function in classifier $\mathrm{Cl}$ when it constructs the fitness function. 


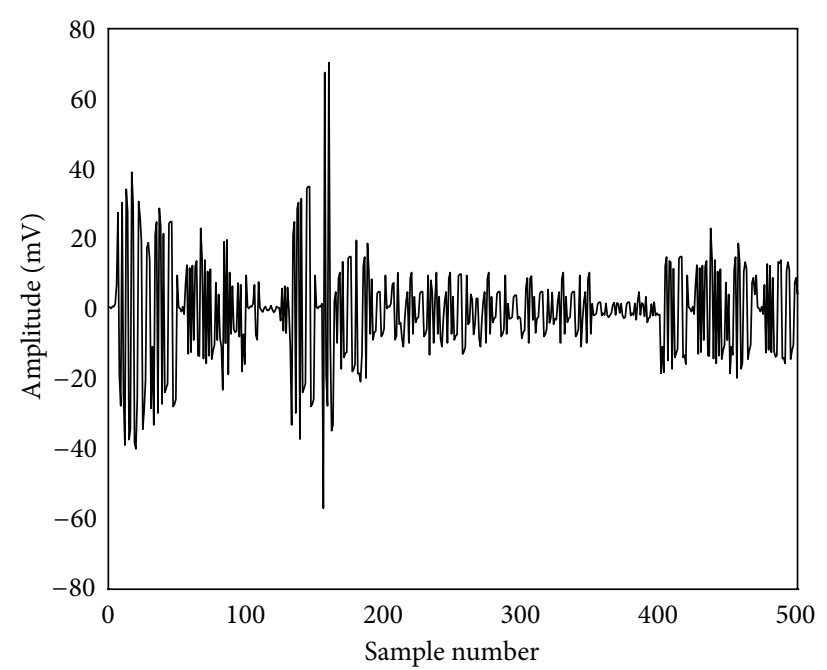

(a) Rock blasting signals

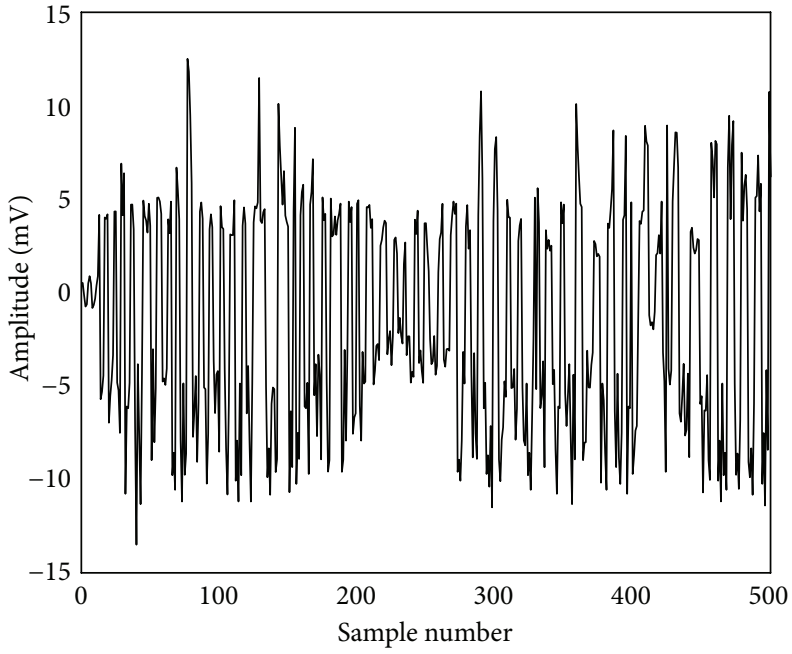

(b) Mechanical vibration signals

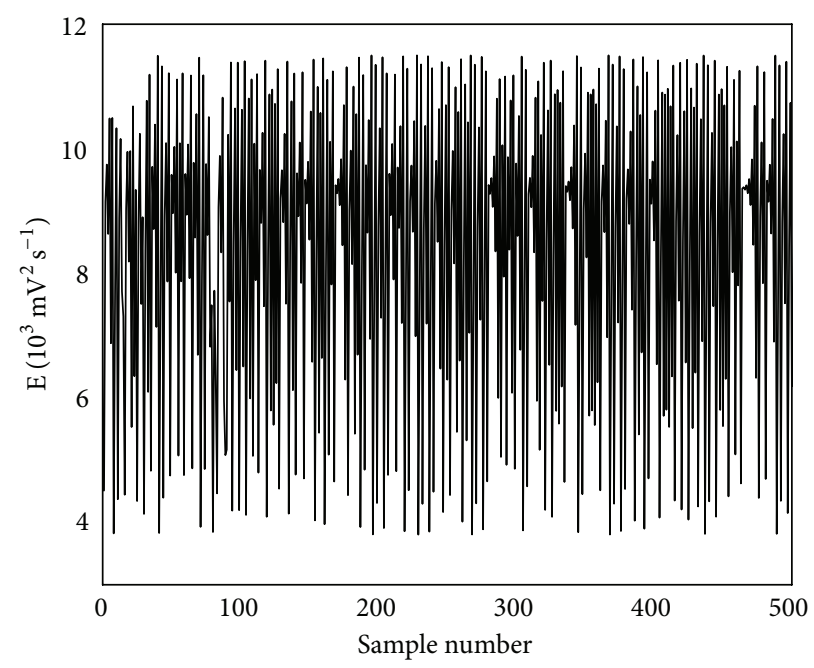

(c) Acoustic emission signals

FIGURE 2: Acoustic emission signals and their interference signals from stope wall rock of underground metal mines.

\section{Conclusions}

(1) Mamdani fuzzy classifier optimized by improved chaos immune algorithm was established and its simulation experimental results showed that the Mamdani fuzzy classifier could effectively improve the prediction accuracy of classification of data sets with noises and outliers.

(2) Mamdani fuzzy classifier based on improved chaos immune algorithm proposed was used for classification and recognition of the acoustic emission signals and interference signals from stope wall rock of underground metal mines. The results showed that the classification accuracy of Mamdani fuzzy classifier based on improved chaos immune algorithm was $90.00 \%$, which achieves to accurately diagnose the acoustic emission signals and interference signals from stope wall rock of underground metal mines.

\section{Conflict of Interests}

The authors declare that there was no conflict of interests regarding the publishing of this paper.

\section{Acknowledgments}

The authors would like to acknowledge Project (51274250) supported by the National Natural Science Foundation of China and Project (2012BAK09B02-05) supported by National “Twelfth Five-Year" Science \& Technology Support Plan.

\section{References}

[1] H. Y. Zuo, Analysis on Mining Safety Mechanism of Underground Metal Mine and Its Disaster Intelligent Prediction, Central South University, Changsha, China, 2012, (Chinese). 
[2] S. K. Palei and S. K. Das, "Sensitivity analysis of support safety factor for predicting the effects of contributing parameters on roof falls in underground coal mines," International Journal of Coal Geology, vol. 75, no. 4, pp. 241-247, 2008.

[3] S. K. Palei and S. K. Das, "Logistic regression model for prediction of roof fall risks in bord and pillar workings in coal mines: an approach," Safety Science, vol. 47, no. 1, pp. 88-96, 2009.

[4] H. T. Liu, N. J. Ma, P. N. Gong, C. L. Zhang, and H. Zhao, "The technology research about hidden danger identification of tunnel roof fall," Procedia Engineering, vol. 26, pp. 1220-1224, 2011.

[5] S. E. Phillipson, "Texture, mineralogy, and rock strength in horizontal stress-related coal mine roof falls," International Journal of Coal Geology, vol. 75, no. 3, pp. 175-184, 2008.

[6] J. Maiti and V. V. Khanzode, "Development of a relative risk model for roof and side fall fatal accidents in underground coal mines in India," Safety Science, vol. 47, no. 8, pp. 1068-1076, 2009.

[7] T. G. Bobick, E. A. McKenzie Jr., and T. Y. Kau, "Evaluation of guardrail systems for preventing falls through roof and floor holes," Journal of Safety Research, vol. 41, no. 3, pp. 203-211, 2010.

[8] C. A. Bertoncini and M. K. Hinders, "Fuzzy classification of roof fall predictors in microseismic monitoring," Measurement: Journal of the International Measurement Confederation, vol. 43, no. 10, pp. 1690-1701, 2010.

[9] M. G. Zheng and S. J. Cai, "An intelligent system for calculating the scale of rational, enlarged production of an underground non-ferrous metal mine," Journal of China University of Mining and Technology, vol. 18, no. 2, pp. 214-219, 2008.

[10] M. M. Asad and R. Dimitrakopoulos, "Optimal production scale of open pit mining operations with uncertain metal supply and long-term stockpiles," Resources Policy, vol. 37, no. 1, pp. 8189, 2012.

[11] H. Y. Zuo, Z. Q. Luo, J. L. Guan, and Y. W. Wang, "Identification on rock and soil parameters for vibration drilling rock in metal mine based on fuzzy least square support vector machine," Journal of Central South University, vol. 21, no. 3, pp. 1085-1090, 2014.

[12] H. L. Fu and Y. F. Sang, "Application of acoustic emission technology to predict roof caving," Chinese Journal of Rock Mechanics and Engineering, vol. 15, no. 2, pp. 109-114, 1996 (Chinese).

[13] Y. N. Wang, C. S. Li, and Y. Zuo, "A selection model for optimal fuzzy clustering algorithm and number of clusters based on competitive comprehensive fuzzy evaluation," IEEE Transactions on Fuzzy Systems, vol. 17, no. 3, pp. 568-577, 2009.

[14] C. S. Li, The Research on the Combination of Fuzzy Clusterings and Its Application, Hunan University, Changsha, China, 2010, (Chinese).

[15] J. Q. E, C. Qian, T. Liu, and G. Liu, "Research on the vibration characteristics of the new type of passive super static vibratory platform based on the multiobjective parameter optimization," Advances in Mechanical Engineering, vol. 2014, Article ID 569289, 8 pages, 2014.

[16] J. Q. E, C. H. Wang, Y. N. Wang, and J. Gong, "A new adaptive mutative scale chaos optimization algorithm and its application," Journal of Control Theory and Applications, vol. 6, no. 2, pp. 141-145, 2008 .

[17] H. J. Wang, J. Q. E, and F. Q. Deng, "A novel adaptive mutative scale optimization algorithm based on chaos genetic method and its optimization efficiency evaluation," Journal of Central South University, vol. 19, no. 9, pp. 2554-2560, 2012.

[18] Z. Y. Xing, Y. L. Hou, and L. M. Jia, "Design of multi-objective genetic-based fuzzy classification system," Journal of Southeast University (Natural Science Edition), vol. 36, no. 5, pp. 725-731, 2006 (Chinese). 


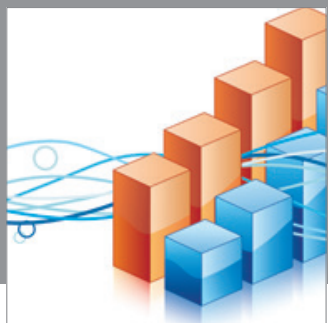

Advances in

Operations Research

mansans

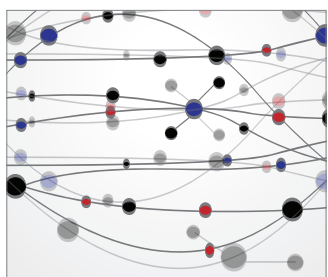

The Scientific World Journal
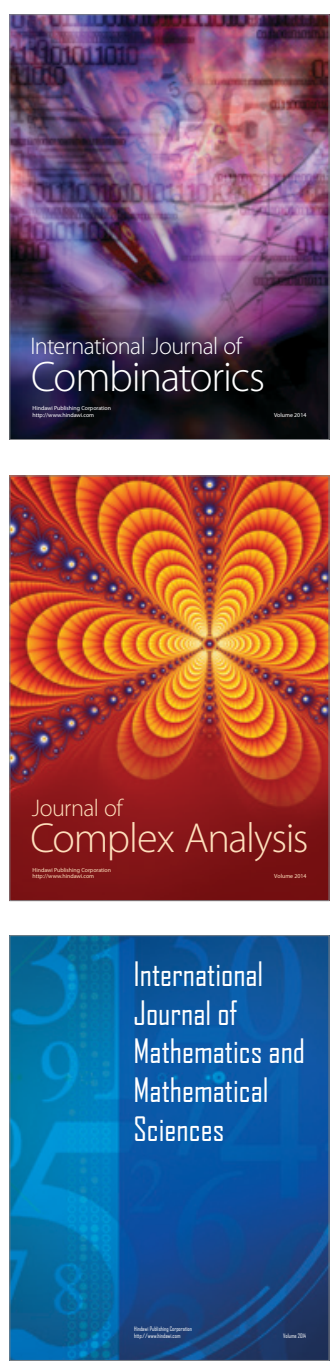
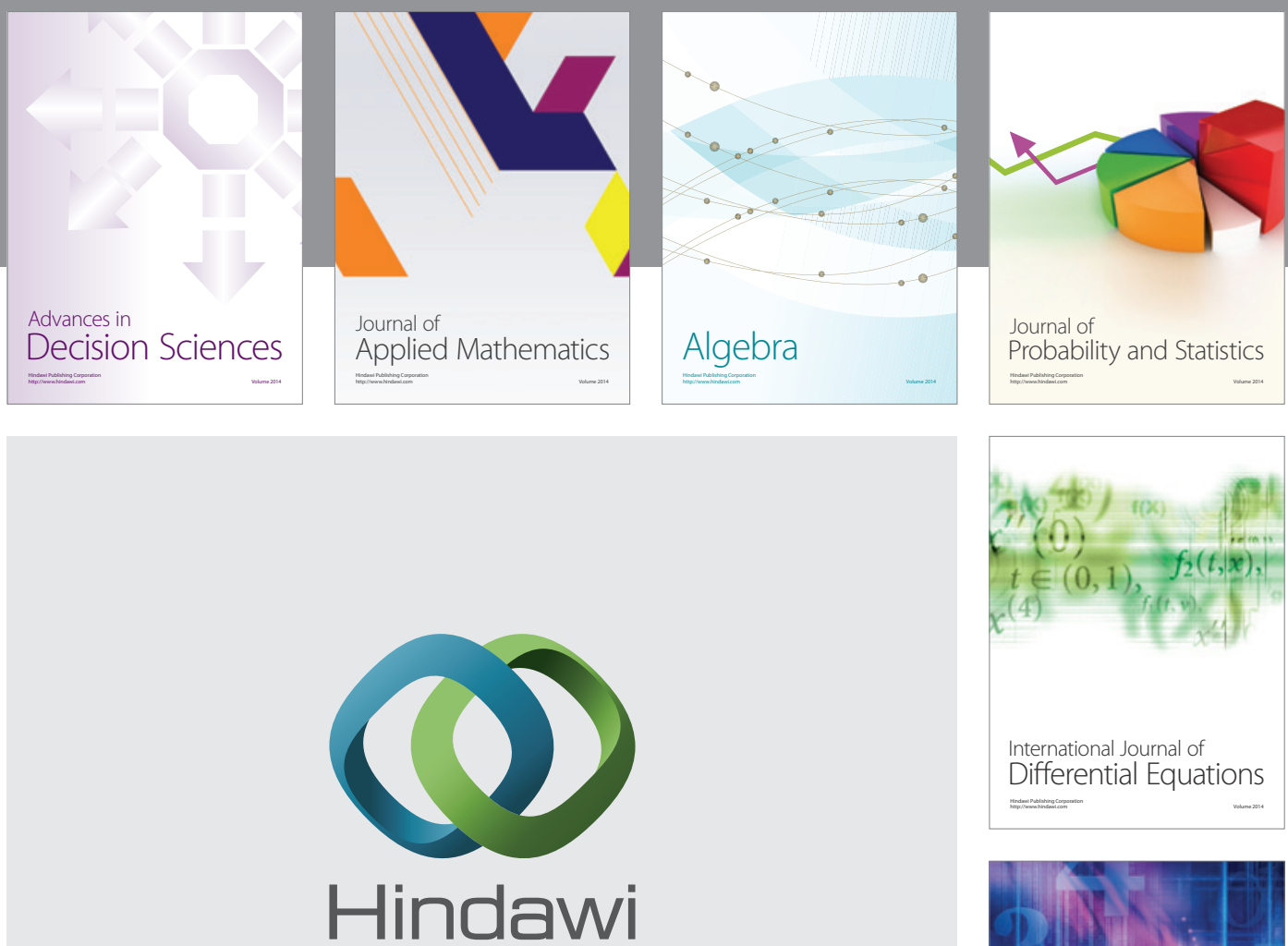

Submit your manuscripts at http://www.hindawi.com
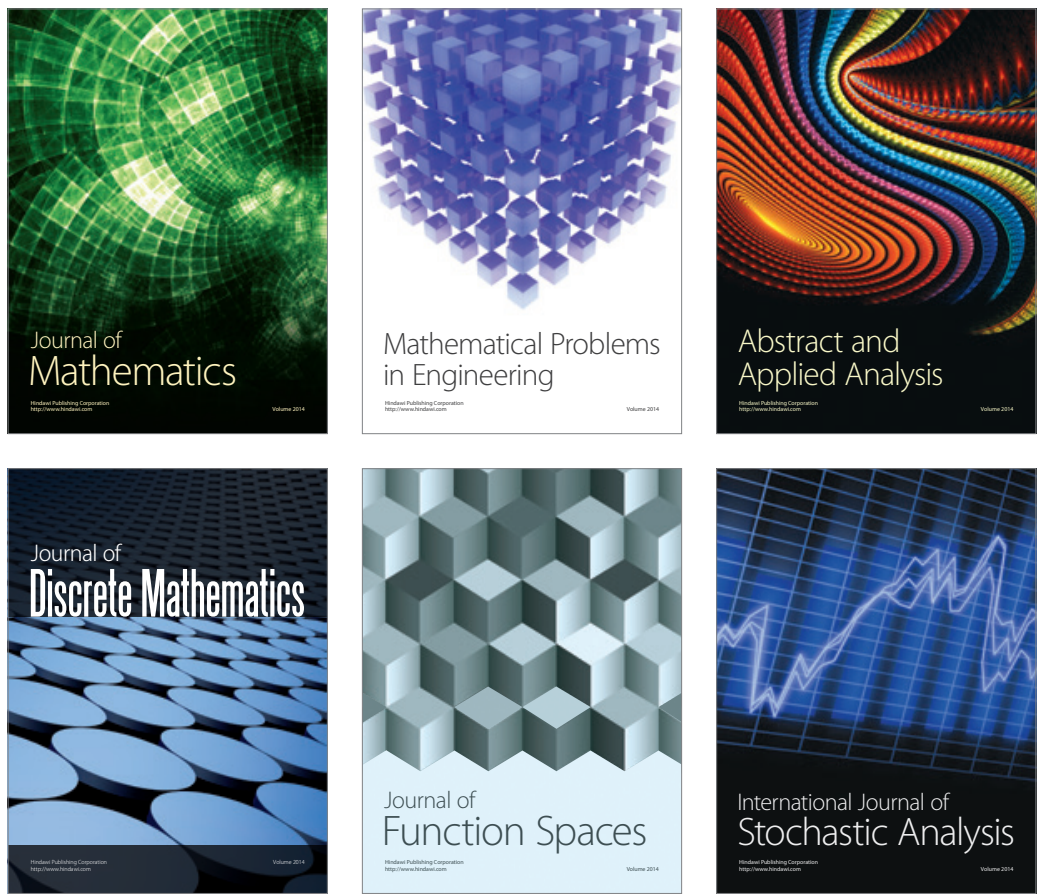

Journal of

Function Spaces

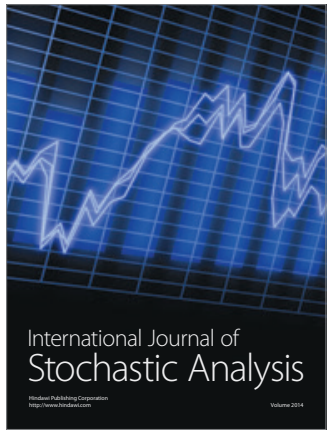

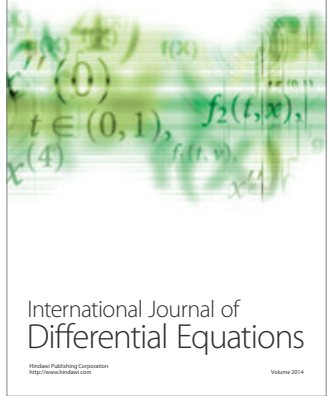
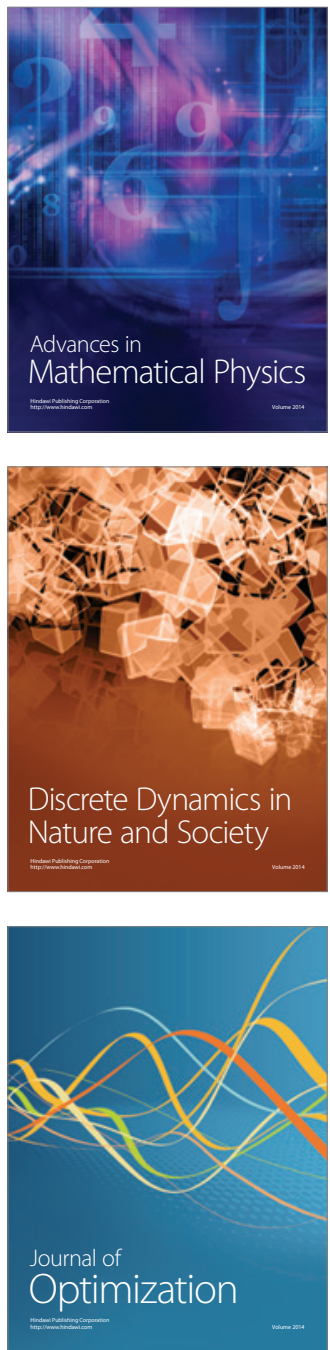